\title{
E-Portals are Valuable Productivity Multipliers, Important Shortfalls in the Safeer System in KSA and Proposed Possible Solutions for Them
}

\author{
Mohamad Najem Najem ${ }^{1}$, Fahad A. Alnoeim ${ }^{1}$, Hisham Najem² \\ ${ }^{1}$ Saudi Culture Bureau, Ottawa, Canada \\ ${ }^{2}$ University of Ottawa, Canada
}

\begin{abstract}
E-portal systems play a big role in various countries. They can be deployed by governments and organizations to provide information for citizens and customers and serve their transactions. Consequently, e-portals can be considered as systems that increase productivity of staffs and offer more reliable services. However, many issues have to be solved, such as: privacy and security, performance, reliability, effectiveness, and documents integrity and archiving. We postulate that once the knowhow is acquired for designing and developing one e-portal system, it then becomes readily conducted and adopted by others. In this work, we examine one such e-portal system that is the Safeer system that was designed and developed for the Ministry of Higher Education (MOHE) in the Kingdom of Saudi Arabia (KSA). We believe that this e-portal system could be reconfigured to serve other sectors, such as agriculture, export and import, fisheries, etc. However, before such reconfigurable deployments is possible, it is necessary first to address the shortfalls and gaps that exist in it and could hinder its deployment for other applications. Accordingly, we have examined the Safeer system and identified a possible set of gaps and shortfalls in it. Of these, we have isolated the most three important shortfalls and proposed possible solutions for them. The three shortfalls are: the study plan or the scheduling, the lacking of integrated email server and services, and the lacking of effective integrated archiving system. We believe that the proposed solutions augment the Safeer portal with additional capabilities, and improve its performance, effectiveness, security and privacy, and data and documents integrity; hence, MOHE would be able to license or sell this product. Moreover, we are confident that this work may be of benefit to others in-charge of planning and discharging e-services to their clients. Finally, we conclude this work and establish a set of possible future directions.
\end{abstract}

\section{Introduction}

Knowledge explosion and information technology age brought by the Internet aroused the interest of governments worldwide to look at an easy way in providing services and information for their citizens [1].
E-government emerged in the early 1990s [2]. Egovernment is the use of technology, particularly web-based Internet applications, to enhance the access to, and delivery of, government information and service to citizens, business partners, employees, other agencies and government entities [3].

With the rapid increase in the use of the Internet as a medium of transaction, government websites quickly evolved into a channel for supporting frontoffice and back-office services, activities, applications and also for providing online services.

Later on, upon realizing the potential of the Internet, individual ministries in various countries began to develop innovative ways to transform their websites into "portals". A Portal is a web based application that offers single point of access to a wide variety of content, data, applications, and services throughout an enterprise [4]. A portal system provides: user personalization, back end customization, single unified sign on point, content aggregation, and portlet component concept. Moreover, portals provide also valuable functions like security, search, collaboration, archiving, and workflow.

Portals have become the preferred web interface used by organizations, institutions, and ministries to provide services and up-to-date information to their users and clients [2]. They are replacing firstgeneration intranet websites as a more effective, efficient and flexible means of managing and delivering applications and information to diverse sets of users. Also, they are potential tools for increasing productivity by enhancing access to an enterprise's information assets [5].

Based on the advancements in the Internet and Information Technology (IT) and the increase in the use of such technologies around the world and specifically in the Kingdom of Saudi Arabia (KSA), and applying the KSA Council of Ministers Resolution no. 40 which deals with e-government implementation rules [6], the Ministry of Higher Education (MOHE) has sought to utilize IT at services she provides. This culminated in the deployment of Safeer portal.

Safeer is a portal that was developed and deployed by MOHE to cater for the needs of all Saudi scholarship students and to facilitate their electronic communication with MOHE and Saudi 
Arabia Culture Bureaus (SACBs). Saudi scholarship students can use Safeer to submit their various requests for the purpose of applying for any of the Academic, Administrative, Personal, and Financial services. The total number of available requests for scholarship students varies from one SACB to another, based on the number of students who are studying at that country. Safeer can make about Eighty Six possible different requests available. Safeer was launched on Sunday 29th of January2009 to link MOHE in the KSA to the Saudi Arabia Cultural Missions (SACMs) around the world. Safeer and its sub-programs were designed according to the latest Information Communications Technology (ICT) international standards [7].

The Safeer system is mainly comprised of four key integrated systems (components). These four components are:

- The Safeer Financial (SF),

- The Safeer Students (SS),

- The Safeer Workflow (SW), and

- The Safeer Academic (SA).

Figure 1 shows an abstract representation of the Safeer system. It also indicates the possible interactions between the four components.

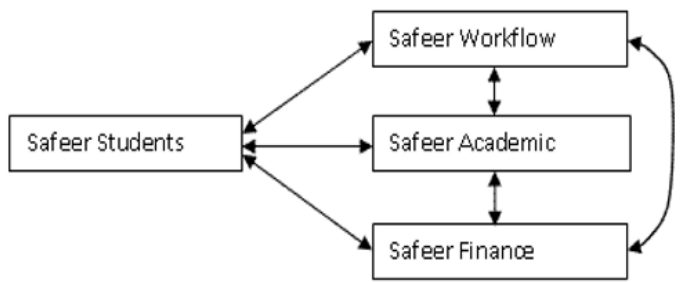

Figure 1. Main components of the Safeer system

- Safeer Students system: aims at enabling students present in various countries around the world to submit their various requests, amend personal data, follow up a service, and conduct many of the applications pertaining to scholarships.

- Safeer Workflow system: aims at raising work efficiency of SACBs and MOHE staff and facilitating the management of students' requests.

- Safeer Financial system: aims at minimizing effort and providing better speed and accuracy at managing financial transactions of both: the large scholarship student count (about 172000 Saudi scholarship students studying at colleges and universities in various countries) and the large staff count that manages, administers, and guides these students and present in the Saudi Arabian Cultural Bureaus (SACBs) in various countries worldwide. Safeer Academic system: aims at maintaining, managing, and tracking of scholarship students' basic details, students' academic progression and academic information.
All these components are important at follow up and service of the Saudi scholarship students, their associates, and their requests. The Safeer portal is capable of performing and hosting integrated services in record time and with high precision. Also, it proved effective for all users whether they are ministry workers, or mission staff, or scholarship students and their associates that are present in various countries.

E-services quality is a key determinant to the success or failure of portal system. E-services quality is the user's perceptions of the outcome of the service delivery along with service recovery perceptions. The factors of e-service quality are: website designs, aesthetic design or visualization, reliability, efficiency, privacy, security, accessibility, performance, and usability $[8,9,10,11]$. With this respect, the Safeer portal has won many prizes for its various features and its advancement in the use of ICT by KSA. An example of such prize is the winning of the ITU prize at World Summit on Information Society (WSIS) which took place in Geneva, Switzerland, in May 2013 [12]. Also, the quality of Safeer portal has been evaluated by many professional referees. For instance, in the work carried out in AL-Zuabi and AL-Shaikhli [13], the quality of Safeer portal was heuristically evaluated by seven experts and eight factors. These eight factors are: accessibility, design and visualization, e-services, effectiveness, performance, reliability, security and privacy, and usability. Accordingly, every dimension was evaluated by different number of items on 5 Likert scales. The evaluation instrument was the survey developed by the researchers upon the reviewed literature. It was found that the Safeer portal quality is fairly good by $75 \%$ and it needs further improvement and development by $25 \%$. However, the researchers recommend further investigation on empirical surveys in order to develop more acceptable and authentic instrument. Also, it was raised that the evaluators need further understanding of the Safeer portal. The evaluators' uncertainty is an ambiguity issue raised in the difference between internal consistency of the survey and the lack of their concordance.

Having stated the above, our research objective in this work is to identify a set of gaps and shortfalls in the Safeer portal system and to provide urgent solutions to some of the most important problems. This is necessary and required in order to improve the effectiveness, reliability, data and document integrity, performance, security and privacy, and to enable the Safeer portal to be configured and deployed for serving other purposes. As a consequence we list the various identified possible gaps and shortfalls, but concentrate only on examining three important issues and proposing possible solutions them; addressing both the backend 
services and the necessary frontend provisions for them. The three shortfalls are:

- The shortfall regarding the study plan services, i.e., the lack of proper scheduling process in the portal system. This service mainly exists in the Safeer Academic system but it should be affected by the interaction of the Safeer Workflow and the Safeer Academic. The study plan is related to factors such as: the efficiency and the performance of the portal system, and also to the productivity of staff using the portal.

- The lack of email services (backend server), which is mainly related to security and privacy in carrying out such email transactions outside vs. inside the portal.

- The lack of an integrated effective archiving service (front end and backend service), which is related to accessibility, effectiveness, data and document integrity, and reliability factors.

We organize this paper as follows: first we have already stated above the abstract and also presented in section 1 the introduction. In section 2, we list the various gaps and shortfalls in the Safeer portal system. In section 3, we discuss the study plan and examine its proposed solution. In section 4, we examine the lacking of the email service and discuss its possible proposed solution. In section 5, we address the lacking of a proper archiving application and present its possible proposed solution. Finally we conclude this work.

\section{Gaps and shortfalls in the Safeer system}

We have been in direct use of the Safeer portal system for more than two years and have suggested many improvements on it. However, the lack of coherence, proper communication channels with MOHE, and slowness in acting on such suggestions triggered us to the writing of this paper so MOHE and others might benefit from it. We first list the various gaps and shortfalls, and then present other concerns. The identified gaps and shortfalls are:

- The portal system does not provide provisions for users with special needs like visual impairment, or audio impairment.

- No integrated internal web browser.

- No provisions for communication channels among users; chat, and discussion board.

- Some services outputs are not delivered to the user without requiring a physical visit to the government office.

- Portal pages are not always downloaded in efficient time.

- No column for notes is available in the Safeer Workflow that indicates the kind of the request that has been processed, only the name of the request is provided.
- No minimal or basic text formatting is supported for formatting the notes that are written by either the students of the staff in SACBs or MOHE. Introducing such minimal formatting would improve readability of the notes and enhance the visual interface.

- No provision for adopting electronic signature scheme is available in Safeer portal. This could be mainly beneficial in the Academic Safeer system, whereby the signature could be applied on issued financial guarantees.

- Orders that are issued by SACBs staff for initiating the releases of financial allowances for students are carried out via separate software applications outside the portal called "Tamid Issuing" applications.

- The link between scholarship students and their associates (e.g., husbands, brothers, etc), who also may become later as scholarship students, is currently done manually and it is not clearly identified. This leads to many problems and a provision for introducing a visual link between them would be of great importance.

- Decisions and applications are carried out via a separate application called the "Administrative Electronic Communication System". This system should be integrated in the portal so that issues and decisions related to students, staff, and authorities could be archived and retrieved through a single point.

- A shortfall regarding the design and implementation of proper study plan service (i.e., the lack of a proper roadmap, or a scheduling process) in the portal. Thus, there is no match between the real roadmaps of the students and their study plans and there is no link between actions on requests $n$ the Safeer Workflow and the manifestation of these actions in the Safeer Academics.

- Lack of integrated email services which affect security and privacy issue with regard to all levels of parties that use the Safeer portal system, included: the staff of SACBs, and MOHE, and also the clients (mainly the students).

- Lack of proper integrated archiving services. This affects data and document integrity, proper storage of information, indexing, and retrieval of information.

Other issues that might be considered in our opinion as weaknesses in the portal, included:

- The client' visual interface of the Safeer Workflow system especially in the latest version that was introduced into service in March 2014 is mostly designed using menubased approach. It could be beneficial to replace it using a ribbonbased approach. In fact, the ribbon approach 
could be applied in both the Safeer Workflow and the Safeer Academic.

- The organization of various tabs (portlets) on the clients' interface of the Safeer Workflow is designed in unprofessional manner, whereby the labels' texts as they appear beside text boxes and the captions as they appear on their respective portlets are small and awkwardly readable.

- The MOHE logo occupies most of the web pages in both the Safeer Workflow and the Safeer Academic leaving little space for the other elements. $\square$ It is messy to have two main drop menus provided on the same page of the Safeer Workflow through which the same set of requests' categories \{All, Inbox, Late, Waiting for reply, Issued, Accepted, Rejected, Delegated can be selected to be processed by the staff.

- Despite of introducing the Safeer system in the early 2009, there are still many processes that are handled manually using paper work. These could be delegated to be done electronically, thus improving the Safeer system effectiveness.

Having identified a list of possible gaps and shortfalls in the Safeer portal system and raised many concerns, we turn on to addressing the three important shortfalls: the study plan services, the email services, and the archiving services.

\section{The study plan shortfall, a proposed solution}

The main subjects of King Abdullah Scholarship Program (KASP) are students. Thus the input to KASP is a student lacking knowledge and the output is a student gaining knowledge. We can abstract this process as shown in Figure 2.

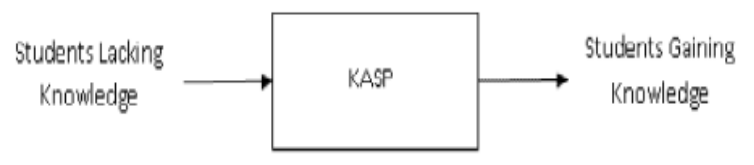

Figure 2. KASP abstracted as a system

The various processes in the KASP system were discussed in a separate paper published by us in the International Journal of Business Strategy (IJBS) [14]. However, the road map of the subject in the real world, which is the scholarship Saudi student, can be mapped and reflected in KASP as a study plan (SP). The study plan comprises the various stages that a scholarship student follows during their studies until he/she fulfill the requirements of his/her degree. For instance, a scholarship student might follow a roadmap that consists of the language learning stage, a qualifying year stage, the master degree stage, and the doctorate degree stage.
Another instance, a scholarship student might follow a road map that consists of a language learning stage, a six month academic period, the bachelorette degree stage, the master degree stage, and the doctorate stage. A third instance, a scholarship student might start with the language learning stage, then postpone his scholarship until he/she get an admission into an academic program. Then the student may start the academic program, say a master degree program, and later extends the scholarship in order to fulfill the requirements of his/her master degree. Then, the student may wait to get admission for a Ph.D. program, then applies to upgrade his/her scholarship to the Ph.D. program. If approved, the student starts a new $\mathrm{Ph} . \mathrm{D}$. stage, then extend his/her scholarship until he/she fulfills the requirements of the degree, and finally applies for ending his/her scholarship and return to KSA. Figure 3 shows sketched examples of a set of presumed road maps.

These road maps are manifested in the Safeer Academic system by a study plan. The study plan is currently created manually and delivered in a table that indicates: the study plan type or status (ended, has not started yet, current, postponed, continued), the start date of it, the program, the field of study, and the name of the university. There is no consistency between the real life map and the created study plan in the system. In that, usually there is only one study plan that is provided and related to the last stage. Thus, valuable information about the previous stages and the road map of the student is lost. Furthermore, since the study plan is created manually, there is no connection between the Safeer Workflow and the Safeer Academic in relation to the study plan.

In addition to the above, the user interface to the study plan is rudimentary and does not enable the user to view the various stages of it. Also, the interface does not provide the means to establish the link between the various requests of the Workflow and the various stages of the study plan. Thus, we believe that the study plan should be created automatically in the portal allowing little manual interference. 


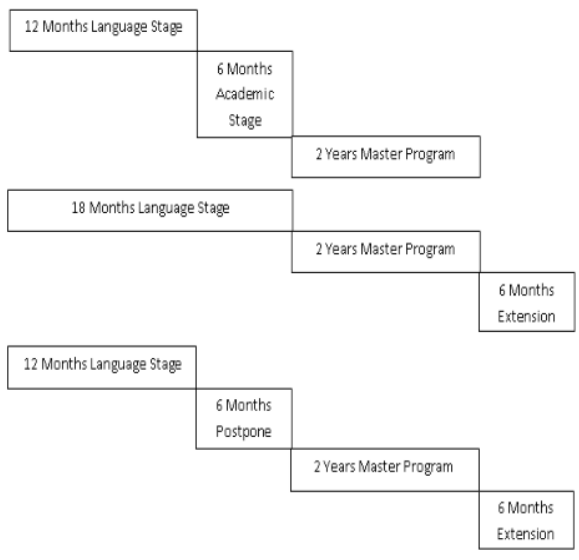

Figure 3. Sketched examples of some road maps

Even though most of the requests are available in the Safeer Workflow (SW) that could enable automatic creation of the study plan, some provisions are necessary to adjust some of these requests and provide added ones. Table 1 summarizes the requests that exist currently in the Safeer Workflow [12], which are not active with respect to the study plan (SP). Apparently, there is no link between them and the study plan. That is currently in the Safeer system there is no link between the Safer Workflow and the Safeer Academic components with respect to the study plan. Hence, the execution/approval of anyone of these requests in the Safeer Workflow does not affect the study plan in the Safeer Academic.

Table 1. Requests in SW, but inactive to the SP

\begin{tabular}{l} 
Requests that are currently available in the Safeer Workflow and may have direct \\
effect on the study plan but are not active in any way with relation to the study plan \\
Open File and Start of Language Program or Academic Program \\
General Purpose Request to transfer from Language to Academic, or to Start of a final \\
Academic Program \\
\hline Postpone in for: Language Program | Academic Program | Previous Period \\
Extend: Language Stage (or Continue Language Stage) | Academic Stage \\
Change of University \\
\hline Change of Specialization \\
Modify End of Scholarship \\
Upgrade: Bachelorette to Master | Master to Doctorate \\
Moving to Another Country \\
End of Scholarship
\end{tabular}

Our objective is to make the above requests active with respect to the study plan and be involved in the automatic creation of the study plan. In order to achieve that, the above requests should be slightly redesigned and reconfigured to enable an automatic creation of the various stages of the study plan. Accordingly, each section of the study plan is created once its respective request is approved in the Safeer Workflow.

Table 2 lists some of the above requests and shows how they should be redone in order to cater for the automatic creation of the various stages of the study plan. Moreover, Table 2 lists new additional requests that have to be introduced in the Safeer
Workflow and have to be implemented to participate in the automatic creation of the study plan. It should be noted that the "General Purpose Request" is eliminated from this list and replaced with a new request called Open Academic Stage. However, eliminating the General Purpose Request from this list does not mean in anyway eliminating it from the requests pool of the Safeer system. The General Purpose Request is still there because it has other usages.

Table 2. New and redone requests in SW to enable automatic SP creation

Requests restructured in the Safeer Wrikflow and some new requests are also introduced in it in order to be used in automatic creation of the study plan

Open File to Start: Language Stage $\mid$ Acade mic Stage

Open Academic Stage: Bridging | Courses | Pre | Qualifying | Bachelorette | Master | Doctorate

Wait for Academic Admission: Courses | Pre | Qualifying | Bachelorette | Master | Doctorate $\mid$ to Upgrade

Postpone ((in) | for): (Language Stage | Acade mic Stage) | Waiting Stage-Not for Upgrade

Resume Studies After Postpone: ((in) (language Stage | Acade mic Stage)

Extend: Language Stage (or Continue Language Stage) | Academic Stage

Change of University (new start | continue program): only affects the study plan if it is approved either in Bachelore tte | Master | Doc torate Stages

Change of Specialization: only affects the study plan if it is approved in either Bachelorette $\mid$ Master $\mid$ Doc torate Stages

Modify End of Scholarship (only affects the study plan if it is approved in either Bache lorette $\mid$ Master $\mid$ Doctorate Stages, otherwise it only changes the ending date of the scholarship to a new one))

Upgrade: Bachelorette to Master | Master to Doc torate

Move to Another Country

End of Scholarship

Each request that participates in creating a new stage in the study plan has dual roles. The first role is to change the status of the previous stage of the study plan from Current to Ended and then to put that stage inactive and make it to appear as dimmed. The second role is to initiate the new stage of the study plan, assign its status as Current, make it appear bright, and designates itself as the annotators.

In addition to the above changes on the requests, the user interface with relation to the study plan in the Safeer Academic system has to be changed to enable the construction of a visual study plan that shows the various stages of it. These stages are constructed with relation to the requests that have participated in creating them. This can be manifested by showing the request number and its type over its respective piecewise or section of the constructed study plan. Also, each section of the study plan is annotated with its time-length (either in days/or months). Figure 4 shows the proposed study plan client interface and presents an example of the visual outputs of this interface. 


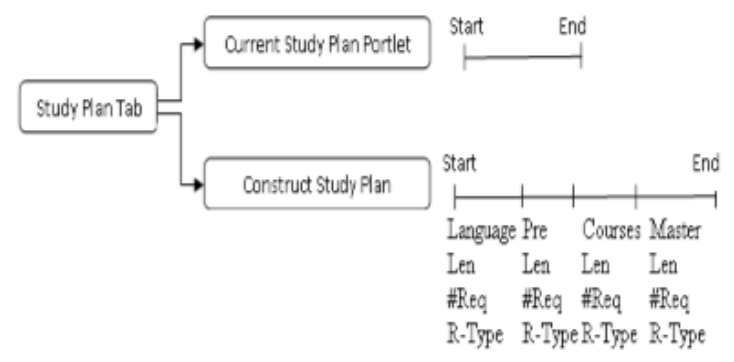

Figure 4. The SP interface, an example of the visual outputs of this interface

\subsection{Benefits of having automatic creation of the study plan in the Safeer system}

- Visual vs. text data tracking and data gathering about the history of a student.

- Efficiency and time saving; searching many requests for collecting information vs. viewing one item which is the study plan that shows the history and various stages for a student.

- More available bandwidth; as a consequence of reducing the amount of search for data related to the history of various students.

- Time saving; in relation to various employees at SACBS and MOHE.

- Increase in performance of the portal.

- Consistency between the real world road map and the study plan; in real world the product is a knowledgeable student manifested by the road map and reflected by the study plan in the Safeer Academic.

- Ability to deploy the Safeer system in other situation that might include a pre-scheduling scheme.

\section{The email shortfall and its proposed solution}

Since the Safeer portal deals with KASP, this requires various channels of communications between individuals and individuals, individuals and institutions, and institutions and institutions.

We first point out that Safeer portal does not offer electronic integrated communication channels such as chat, discussion board, and an integrated email services among users at various levels. However, the main electronic communication channel is external email services and it is used to convey to the students the various decisions that are made by the various employees on the submitted requests, or to inform them about any procedure that has been implemented on them, such as issuing a warning, or issuing a salary stopping. It is also used by the students to respond to certain inquiry emails that were issued from within requests in the Safeer Workflow. Moreover, it is used by workers from within the Safeer Academic to send some information regarding certain issues such as deadlines to submit a study report, or a study plan.

Even though privacy and security is important in Safeer portal architecture, the email service is left unattended for and to be carried out between SACBs and the students and between MOHE and the students through general free email services, such as: Gmail, Outlook.com, hotmail.com, ICloud, Yahoo, Zoho, AIM, Mail.com, Shortmail, Hushmail, and Inbox.com. Refer to [15] to get a list of free email services.

Leaving the only electronic communication channel with students under the mercy of free email services providers jeopardizes the security and privacy notion in the Safeer system. Students do communicate valuable information through their emails, including their personal documents, their Saudi Identities, their academic records, documents related to their dependents and families, bank accounts, and other documents related to their various activities. These emails could be read by staff of the email services providers, can be hacked and subject to identity theft, and can be released by these service providers to other parties without consulting their clients for obtaining their permissions. Thence, the privacy of email users of the Safeer system should be given a top priority, be respected and protected. Refer to [16] for further information about email security and privacy issues. Consequently, it is necessary to provide provisions for email privacy in Safeer system.

We propose that an email services should be integrated into Safeer system. Once a student is accepted in KASP an email account should be created for him/her, similar to the procedure that is followed in various universities.

To unify login manner, an email account consists of user identification which is the Saudi Identity number plus a password that consists of at least 8 characters constituted of lower, upper, special, and numerical letters. Consequently, all documents are saved on the email server at MOHE and not left to be under the penetrations of various email service providers.

For example, MOHE could integrate into Safeer system a free email server such as Microsoft Live@edu email server, or an open source email server such as Zimbra, or could integrate a proprietary email servers such as Microsoft Exchange Server, or Google email server Integrating the email server into Safeer system provides additional capability related to data reliability and integrity in Safeer. The various emails and attached documents, are saved on, and could be retrieved, from the same email server installed at the MOHE site. In addition to that, important emails that are issued between certain authorities could be encrypted using nationally designed algorithms. Other 
advantage of having email services integrated in the Safeer portal is to enable the deployment of Safeer for other organizations where email services should provide very high privacy and security medium of communications.

\subsection{Benefits of integrated email server in the safeer portal}

- Catering for privacy and security issues related to email services.

- Archiving emails and their attached documents in MOHE site.

- Assigning special tasks to specific email addresses.

- Users can be added immediately.

- Unified email experience.

\section{Archiving shortfall and its proposed solution}

Indexing, storage, and retrieval of the vast amount of varied documents related to students and staffs are not a straightforward procedure. A student documents can be arranged into a set of main categories, included:

- Decision and application documents,

- Financial documents,

- Academic documents,

- Supporting documents,

- Personal documents,

- Associates documents,

- Referral documents, and

- Equivalency documents.

Each main category consists of a set of subcategories. For example, the Academic Documents (AD) main category consists of the set of subcategories; $\mathrm{AD}=\{$ Certificates, Study plans, Study reports, Others $\}$. A subcategory consists of a set of individual documents or a set of subcategories. For example, the Certificates subcategory consists of a set of individual certificates; $\mathrm{C}=$ \{language certificate, bachelorette certificate, master certificate $\}$ mean while the Others' subcategory consists of a set of subcategories, such as disciplinary actions and change of supervisors; $\mathrm{O}=$ \{\{Warnings $\}, \quad$ Change of supervisors $\}$, \{Dismissals $\}$ \}

The question that might arise is that why we are addressing the archiving topic? And the answer is manifested in solving most of the problems that are rising in the archiving processes and procedures that are currently followed by SACBs and MOHE. The current archiving mechanism is subject to the following problems:

- Repeated documents.

- Documents are scattered here and there inside or outside their categories.
- Intermix between documents that belong to

- other students become apparent and shows in several electronic files.

- Lack of retrieval and searching technique for specific document; instead of going through all the electronic file of a student in order to locate a specific document.

- The documentation process is carried out through separate systems and includes a lot of manual work which is borne to errors.

- No integrated processes that would enable the integration of the Data Management System (DMS) in the Safeer system. Thus there is no consistency and integrity in the archiving of various documents. There is no proper verification procedure of the archiving professes that should be followed in SACBs and MOHE.

Archiving of various documents related to students is important in order to keep track of various activities during the road map of each student. The electronic file of each student is built up of many sections (i.e., the Categories) and each section is titled according to the type of the documents it contains. Accordingly, the sections are titled as stated in [17] as: Decisions and Applications, Financial, Academic, Supporting, Personal, Associates, Referred, and Equivalency.

The electronic file of each student is populated through the processes of archiving. The archiving processes should be reliable, and preserve data/documents integrity. There are two main avenues for archiving. The first is done through the attached documents in various requests that were submitted by the student through the Safeer Students and admitted to the Safeer Workflow for approvals or refusals. The second avenue is done outside the Saffer system via staffs of SACBs and MOHE in order to archive further documents. These documents are either produced as output documents from Safeer system and to be signed by authorities at SACBs and MOHE, such as for example the Financial Guarantees, or documents that have to be done manually in order to satisfy the outputs of some approved requests, such as for example the Extension of Scholarship Request. Furthermore, it is necessary to archive other that are coming from external sources, such as letters from colleges and universities, documents produced as a consequence of personal visits of students to SACBs and MOHE, etc.

The archiving process that is carried out through requests is more reliable compared to the external archiving process. This is because each student submits his/her/documents attached to the submitted request. However, there are many drawbacks for this avenue. One drawback is that it may lead to repeated archiving of same documents so that the electronic file becomes overpopulated, requires more storage space, and also requires more time to retrieve and 
download. Another drawback is related to scattering of the submitted documents in unrelated categories or subcategories, thus distorting the structure of the electronic file.

The other archiving avenue which is carried manually and outside the Safeer system has also many drawbacks. This is because most of the front end processes are executed manually by the employees at SACBs, and MOHE. Manual processes are subject to human errors and could lead to intermixing of students' documents. Also, printing or stamping incorrect codes on the documents cause scattering of these document all over the electronic files. The documents that could be archived externally and manually by various employees included: financial guarantees, financial guarantees for admission purposes, orders of start/restart finance releases, orders of end of scholarship finance releases, orders for compensations of theses printing, orders of rewards of excellence finance releases, documents annotated with internal decisions at various SACBs and MOHE, documents and letters received from universities, claims and complaints submitted manually by students, and more importantly, students' publications, namely manuscripts and accepted papers in international conferences.

The manual archiving process is carried out using the Barcode technique. For instance, a Barcode SACB Management System is deployed as frontend system and utilized by each employee in SACBs. For example, in SACB-Canada this Barcode SACB Management System is in fact a system that was developed for inventory stock control and has been modified to be employed in the frontend archiving process. In this system the frontend archiving process relies only on the Saudi student national identity number and there is no verification process whatsoever that associates this identity number to the name of the holder. Thus, the integrity of the documentation process is lost and a mix of documents that belongs to different students start to appear in the various electronic files. In Addition to that, the manual archiving process in itself is time and device consuming. A barcode is generated for each document under a transaction that includes the entered Saudi student national number, which has to be printed on the document under processing. Then these bar-coded documents are sent to the archiving unit in SACB and these documents are scanned and introduced in the backend Data Management System (DMS) at MOHE. Mistakes in valid Saudi IDs will go through unnoticed and thus, a bar-coded document might end being inserted in unintended student electronic file or placed in unintended category.

In order to overcome these problems, one way to prevent this from happening is to instruct certain employees in the various archiving units in SACBs and MOHE to visually inspect and review each electronic file and carefully and accurately rearrange the documents and/or to remove these repeated documents it. However, such a process is overwhelming and still error an error source.

\subsection{The proposed archiving solution}

To overcome the above problems, we propose the following possible feasible automatically conducted solution which addresses also the necessary changes to be performed in the Saffer system in order to enable proper, reliable, and documents integrity archiving mechanism.

The necessary changes will take place on both the frontend client side by introducing the Archiving request and on the backend by integrating the DMS application into the Safeer system. In both cases we could still use the Barcode concept as the category and the individual document indexing technique inside a student electronic file. This is considered in order to maintain backward compatibility with previous past archiving processes in which each main category is assigned a different fixed barcode. Instead we could also use a numbering approach where numbers are linked to a predefined set of stored barcodes and eliminate the use of the Barcode SACB Management System and the printing and scanning processes associated with it. The numbers are considered as aliases for the predefined barcodes and are used only to facilitate to the staffs for rearrangement of documents into their appropriate categories and subcategories in the Archiving request

\subsection{Documents indexing technique for storage and retrieval}

Figure 5 shows the general indexing approach for categorizing and indexing of individual documents in a student electronic file. Note that this arrangement is only for illustration, it does not mean the use of sequential file indexing organization approach.

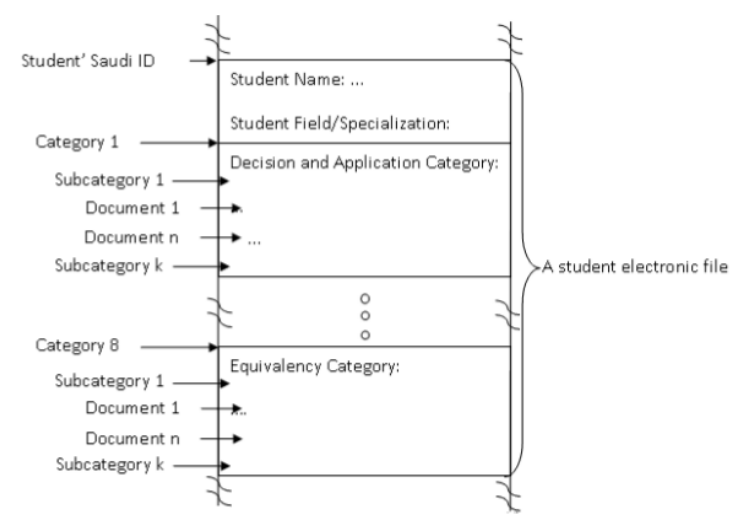

Figure 5. General form of indexing and retrieving documents 
Each electronic file is indexed with the unique student Saudi identity number. Inside the electronic file the reference pointer to a category in a student electronic file is a combination of the student' Saudi ID and the Category' number. Similarly, the reference pointer to a document is a combination of student' Saudi ID, the Category' number, and the document number.

As we already mentioned that it is necessary to introduce changes on both the backend and front end services.

At the backend it is necessary to integrating the Data Management System (DMS) into the Safeer portal system.

At the frontend, first it is necessary to introduce a new request into the Safer Workflow, called Archive request. This request is only available for staffs ar SACBs and MOHE not to the students. Through this request, the appropriate documents can be attached into it and arranged into their various categories or subcategories and then the request is submitted for further verification via another layer of the employees hierarchy, thus, minimizing errors in inserting and allocating documents to their respective categories inside a student electronic file. Second, it is necessary to introduce at each staff terminal a small database that stores the set of predefined category and subcategory barcodes, or alternatively to incorporate this database at the backend DMS application. These barcodes will be used to be electronically stamped on their respective documents. These stamped barcode documents are the ones to be inserted and attached into the Archive request.

The Archive request is created in the Safeer Workflow using the student' Saudi ID-number. Hence, documents can now be verified to belong to their owner, because the name of the student and other information related to him/her appear on the created request. It is also possible to create separate IDs for other purposes such as the archiving of the general decisions and applications related to KASP.

Once the created Archive request has been submitted and approved, then the attached documents to it would be streamed and placed into their appropriate Categories and Subcategories in the student' electronic record.

Table 3 shows the configuration of only the top level structure of the Archiving request. In this configuration only the main documents Categories are shown.

Table 3. Archive request top level configuration Archive request to be introduced in Sajegr Woriffow Archive: Decisions and Applications | Financial | Academic | Supporting | Personal | Associates |Referal| Equiralency|.

The Archive request finds its end route in the Archiving Unit in each SACB or in the Archiving
Unit at MOHE, where the final touches are carried out and the attached documents arranged up to the archiving MOHE standards and finally the request being approved.

In addition to introducing the Archiving request in the Safeer Workflow and the predefined barcode database, it is also necessary to introduce new tabs and portlets into both the Safeer Academic and the Safeer Workflow. This is necessary in order to enable the staff to access the MOHE-DMS in order to retrieve students' electronic files.

\subsection{Benefits of using the proposed archiving mechanism}

- Enables more effective way of archiving.

- Enables verification of the archiving process of documents.

- Eliminates the use of external barcode system and its associated devices.

- More reliable and less error prone technique.

- Enhances documents integrity in archiving.

- Requires less maintenance efforts and time.

- Enables Safeer system to be deployed in situations where automatic archiving of documents is required. Introduces flexibility of the use of DMS by staffs.

\section{Conclusions and future work}

Portal systems are valuable tools in various countries. Especially, they could play a big role in the developed countries, because they allow for the introduction of new technologies and the increase of workers productivity. Safeer system is an important portal that was developed in KSA to serve MOHE, SACBs and the Saudi scholarship students. Improvements in this portal could enable its deployment to be used for other purposes. However, it is necessary first to overcome any shortfalls and gaps in it. Therefore, in this paper we have pointed out many gaps and shortfalls that are associated with the Safeer system and addressed only the three important ones. These three shortfalls are the study plan, the email services, and the archiving services. We believe that if the other remaining gaps where rectified, then the quality of the Safeer portal will be enhanced and several versatile deployments of the Safeer portal become feasible. However, despite all these gaps, we could state that he Safeer portal is fairly good and has much strength that we did not dwell on, because they are out of the scope of this work. We could conclude that Safeer portal at the end offers a good service for scholarship Saudi students, MOHE, and SACBs. However, as further improvements and developments introduced in the Safeer portal, further evaluations of its quality and its comparisons with other portals is needed to be done. 
We recommend that the evaluation process of Safeer portal should be carried out by both experts and ordinary users. Also, it is necessary to measure the effectiveness, performance and degree of sophistication of the Safeer portal to other portals and mainly to other e-government portals deployed in Saudi Arabia and if it is possible to other portals deployed in other countries. Finally, it is necessary to measure how far the enhanced Safeer portal would penetrate the market after mending the various gaps in it.

\section{References}

[1] H. Al-zuabi and M. Mahmud, "Implementation of egovernment in Arab countries: A Literature review". International Conference ICRIIS, Malaysia, 2011.

[2] B. Maheshwari, V. Kumar, U. Kumar, and V. Sharan, "Framework for e-government portal development". In Egovernment development and diffusion: inhibitors and facilitators of digital democracy, Hershey, PA, 2010, Information Science Reference.

[3] G. N. L. Stowers, "Commerce Comes to Government on the Desktop: E-Commerce Applications in the Public Sector". The PricewaterhouseCoopers Endowment for the Business of Government, StowersReport.pdf, U.S.A, 2001. http://www.businessofgovernment.org/sites/default/fil es/StowersReport.pdf.

[4] G. B. Shelly, A. Napier and A. H. Rivers, "Discovering the Internet: Complete Concepts and Techniques", 4th edition, Cengage Learning, 2012. ISBN: 13: 9781111820725 .

[5] P. B. Van, "Information portals: a strategy for importing external content". Electronic Library, vol. 21, no. 16,2003 , pp. 591-600.

[6] MCIT, "ICT indicators in K.S.A". Ministry of Communication and Information Technology, 2009, http://www.mcit.gov.sa/english.

[7] SACB-Australia, News Archive, SAFEER Program Receives International Telecommunication Union's (ITU) Prize, awarded at the World Summit on the Information Society (WSIS) which took place in Geneva, Switzerland in May 2013. http://www.sacm.org.au/E/news/4.html.

[8] M. A. Alanezi, A. Kamil and S. Basri, "A proposed instrument dimensions for measuring e-government service quality". International Journal of u- and e- Service, Science and Technology, vol. 3, no. 4, 2010, pp. 1-18.

[9] J. -H. Kim, M. Kim and J. Kandampully, "Buying environment characteristics in the context of eservice", European Journal of Marketing, vol. 43, no. 9/10, 2009, pp. 1188-1204.
[10] R. P. Bringula and R. S. Basa, "Factors Affecting Faculty Web Portal Usability". Educational Technology \& Society, vol. 14, no. 4, 2011, pp. 253- 265. http://www.ifets.info/journals/14_4/21.pdf.

[11] Z. Yang, S. Cai, Z. Zhou and N. Zhou, "Development and validation of an instrument to measure user perceived service quality of information presenting web portals". In Information \& Management, vol. 42, 2005, pp. 575-589.

[12] SACB. "Saudi Scholarship Guide and Electronic Requests", 2014. http://www.saudibureau.org/.

[13] H. F. Al-zuabi and I. F. Al-shaikhli, "Quality Evaluation of "Safeer" Portal for Saudi Students Studying Abroad". International Conference on Advanced Computer Science Applications and Technologies ACSAT2012, 26th to 28th, Kuala Lumpur, Malaysia, 2012, Also appears as DOI 10.1109/ACSAT.2012.92.http://www.academia.edu/ $3987769 /$.

[14] F. A. Alnoeim and M. N. Najem, "A Proposed Strategy Framework for the King Abdullah's Scholarship Program". International Journal of Strategic Management, IJSM, vol 14, number 1, 2014, pp. 7-34.

[15] H. Tschabitscher, "Top 11 Free Email Services". 2014. http://email.about.com/od/freeemailreviews/tp/ free_e mail.htm.

[16] T. Wright, "Privacy Protection Principles for Electronic Mail Systems". Information and Privacy Commissioner Ontario, 1994.http://www.ipc.on.ca/ images/Resources/email-e.pdf.

[17] MOHE, "Document Management System (DMS)", 2014. http://dms.mohe.gov.sa/mohe/Login.aspx. 